\title{
Linx
}

Revue des linguistes de l'université Paris X Nanterre

$53 \mid 2005$

Le semi-figement

\section{La source de 'l'approximation' dans par ici, par là, par là-bas}

\section{Yukiyo Homma}

\section{(2) OpenEdition}

Journals

Édition électronique

URL : http://journals.openedition.org/linx/269

DOI : $10.4000 / \operatorname{lin} \times .269$

ISSN : 2118-9692

Éditeur

Presses universitaires de Paris Nanterre

\section{Édition imprimée}

Date de publication : 1 décembre 2005

Pagination : 121-134

ISSN : 0246-8743

\section{Référence électronique}

Yukiyo Homma, «La source de 'l'approximation' dans par ici, par là, par là-bas », Linx [En ligne],

53 | 2005, mis en ligne le 14 février 2011, consulté le 01 mai 2019. URL : http://

journals.openedition.org/linx/269; DOI : 10.4000/linx.269

Département de Sciences du langage, Université Paris Ouest 


\title{
La source de 'l'approximation' dans par ici, par là, par là-bas
}

\author{
Yukiyo Homma \\ Université de Paris $X$ et Laboratoire MoDyCo (CNRS UMR \\ 7114)
}

\section{Introduction}

Notre objectif est de prouver que, dans une expression, même figée ou semifigée, chacun de ses éléments garde son identité. Par « identité », nous entendons une caractérisation abstraite et générale du fonctionnement d'un terme propre à rendre compte de la singularité de chacune des valeurs en contexte de ce terme. Il s'agit de ce que l'école culiolienne appelle «Forme Schématique » (dorénavant, FS), qui est considérée comme sous-tendant tous les emplois d'un terme. Nous allons réfléchir, dans ce présent article, sur les expressions telles que : Il s'amusait par ici / Il est né en 1980 on 1982... par là / On va s'installer par là-bas? Il est significatif dans les énoncés que nous abordons ici que, d'abord, la forme par $Y$ (ici, etc.) exprime une approximation en paraphrasant globalement des termes tels que environ (dans les environs) ou à peu près, et qu'ensuite, cette approximation n'est exprimée que dans le cas de la forme 'par + déictique' : Il s'amusait ici. vs Il s'amusait par ici. Nous traitons donc en bloc la combinaison de par et de ces termes déictiques.

Nous avons effectué nos analyses à partir du point de vue selon lequel une préposition est un relateur entre deux entités X et Y. Ce qui suppose la forme virtuelle 
$X$ prép. $Y$, dans laquelle $\mathrm{X}$ est repéré et $\mathrm{Y}$ est un repère. Une préposition étant un relateur entre $\mathrm{X}$ et $\mathrm{Y}$, l'analyse et la représentation de l'identité d'une préposition doit tenir compte de ces entités ${ }^{1}$. Dans la plupart des cas, Y est représenté par le régime de la préposition, c'est-à-dire par le terme (ou syntagme) qui suit celle-ci. Par contre, il est souvent difficile d'identifier X. Il arrive que X ne soit pas représenté, dans l'énoncé, par un seul terme, par un verbe par exemple. Nous n'allons pas développer cette problématique ici.

Compte tenu de nos objectifs, nous présentons d'abord notre hypothèse sur la FS de par, établie en tenant compte de l'ensemble des emplois de cette préposition ${ }^{2}$.

\section{La FS de par}

\subsection{Hypothèse sur la FS de "par»}

Dans la forme (virtuelle) $X$ par $Y$, $Y$ repère $\mathrm{X}$ de telle sorte que la visée $(\mathrm{P})$ se réalise à travers l'appariement, établi par $\mathrm{Y}$, entre deux entités A1 et A2, qui se codéterminent relativement à $\mathrm{P}$. Cette co-détermination est marquée par la tension oppositionnelle entre A1 et A2 qu'impose la visée P.

Comment comprendre la relation d'appariement entre $A 1$ et $A 2$ ? Prenons comme exemple le cas de pari. L'appariement entre deux entités, à savoir deux choix (A1 et A2) réalise ici la «visée » $(\mathrm{P})$ parier. Ce statut de «constituants » de $\mathrm{P}$ fait que A1 et A2 n'existent que l'un par rapport à l'autre relativement à la visée, donc sont qualitativement discriminatoires en tension.

Pour une meilleure compréhension, illustrons la FS de par en dégageant quatre de ses manifestations dans lesquelles A1 et A2 sont configurés différemment :

$1^{\text {er }}$ cas de figure : le passage entre deux états (de fait) oppositoires (A1 et A2).

Par exemple, dans Jean a été renversé par un camion, la visée P est «le changement d'état de Jean causé par une entité extérieure ». P impose deux états qui se codéterminent, à savoir «l'état de référence de Jean » (A1) et «l'état de Jean subissant le résultat du procès renverser » (A2), c'est-à-dire, l'état qui se distingue de A1. Ici, A2 correspond à X exprimé dans la proposition principale Jean a été renversé. Un camion $(\mathrm{Y})$ est conçu comme entité réalisant $\mathrm{P}$, c'est-à-dire, entité mettant en relation $\mathrm{A} 1$ et $\mathrm{A} 2$.

$2^{\text {ème }}$ cas de figure : $\mathrm{A} 1$ et $\mathrm{A} 2$ sont des occurrences en succession relativement à la visée.

Par exemple, dans Sa journée commence par une lecture, la visée (P) est «le déroulement de sa journée », qui est implicite. Y (une lecture=A1) est une des étapes intégrées dans $\mathrm{P}$, définie par le procès commencer marquant l'étape du début; on

\footnotetext{
${ }^{1}$ Il est important de noter que $\mathrm{X}$ ou $\mathrm{Y}$ ne sont pas toujours explicités dans l'énoncé (ex. A terre ! ; Il faut faire avec) mais nous considérons que ces deux entités sont toujours exprimées par l'énoncé qu'elles soient explicites ou implicites.

${ }^{2}$ Faute de place, nous ne pouvons malheureusement pas démontrer tous les emplois de par dans cet article.
} 
conçoit l'existence d'autres étapes (A2) qui suivent cette première étape. Une étape implique forcément sa relation sémantique avec d'autres étapes tout en occupant un statut irremplaçable par d'autres étapes. Donc, A1 et A2 sont en relation co-déterminatoire tensive.

3ème cas de figure : Y représente A1 ou A2, un des éléments d'une même série.

Par exemple dans passer par Lausanne, Y (Lausanne) est considéré comme un des choix possibles, dans une même série, pour " atteindre le lieu visé » $(\mathrm{P})$, en effectuant le procès passer $(\mathrm{X})$. Dans le cas de l'exemple en question ici, la réalisation de la visée oblige à choisir une seule possibilité en entrainant la relation co-déterminatoire tensive entre « lieu de passage à non valider » (A1) et «lieu de passage à valider» (A2, qui est ici Y). D'où l'interprétation de l'exemple passer par Lausanne comme 'atteindre le lieu visé en passant par Lausanne mais pas par d'autres lieux de passage possibles'.

$4^{\text {ème }}$ cas de figure : $\mathrm{Y}$ représente une estimation subjective, A2, qui se réfère à A1.

Par exemple dans Par un beau matin de printemps, le prince partit en voyage, on " présente la situation comme un moment spécial» ( $\mathrm{P}$, la visée). La situation est dotée d'une qualification particulière, ce qui conditionne cet emploi de par (*Il arriva par jeudi matin / Il arriva par un froid jeudi matin). Ainsi par n'est possible que si Y possède une certaine qualification qui distingue la situation où se produit «le départ du prince en voyage » $(\mathrm{X})$ relativement à des situations conçues comme habituelles ou non particulières - ces dernières constituant la référence (A1) par rapport à laquelle s'opère l'estimation portée sur Y (A2). La co-détermination entre $\mathrm{A} 1$ et $\mathrm{A} 2$ relativement à $\mathrm{P}$ engendre l'interprétation de l'exemple en question comme «Le départ du prince en voyage » $(\mathrm{X})$ s'est effectué dans « une situation qui est spéciale 》 (A2) mais pas dans « une situation habituelle » (A1).

Nous avons explicité quatre cas de figure de la FS de par. Cependant, même pour un même cas de figure, il est difficile d'établir une relation entre l'emploi analysé et d'autres emplois. D'où la nécessité de l'examen détaillé de chacun des emplois.

\subsection{Analyse d'autres cas relevant des quatre types de figure de la FS précé- demment étudiés}

\subsubsection{Premier cas de figure: le passage entre deux états (de fait) oppositoires (A1 et A2).}

\subsubsection{Les expressions du type finir par + infinitif}

L'expression finir partinfinitif est comparable à des expressions du passif (être renversé par un camion) dans la mesure où l'énoncé exprime le passage entre A1 et A2. Observons cependant quelques différences :

\section{Thierry a fini par dévoiler la vérité.}

Y (dévoiler la vérité; plus précisément, le fait que Thierry ait dévoilé la vérité) est représenté comme un état de fait (ou un résultat) contraire à la prévision du locuteur établie compte tenu de l'état de fait initial, à savoir le fait que Thierry ne dévoilait pas 
la vérité. Le locuteur conçoit $\mathrm{Y}$ comme un état de fait qui tient compte de l'état de fait précédent dans un scénario. Y est représenté, par le biais du verbe finir, comme la fin du scénario. L'exemple (1) suppose que Thierry «s'était obstiné à ne pas dévoiler la vérité » (A1) mais est amené à « dévoiler la vérité » ( $\mathrm{Y}$, qui représente ici $\mathrm{A} 2)$ grâce à un certain nombre d'évènements : son entourage a fait pression sur lui ; il ne voulait plus être complice d'un mensonge qui prenait des proportions imprévues, etc. Ainsi, nous pouvons dire qu'un certain nombre d'évènements, qui sont implicitement évoqués, permettent le passage entre A1 et A2, autrement dit, une relation entre eux. Avec l'expression finir par+infinitif, la visée (P) serait «mettre fin au scénario ». Cette visée correspond à $\mathrm{X}$ qui est exprimé dans l'énoncé à travers le verbe finir.

Dans (1), Y représente A2 qui est implicite dans (1), alors qu'avec le passif (être renversé par un camion), $\mathrm{Y}$ (un camion) exprime "le moteur du passage entre $\mathrm{A} 1$ et $\mathrm{A} 2$ », c'est-à-dire l'entité qui permet le passage entre A1 te A2.

\subsubsection{Par miracle, par chance, par bonheur, par malheur}

(2) Par malheur, Jean a raté son baccalauréat.

(3) Par miracle, Pierre a réussi au concours.

Ici, le fait exprimé dans (2) ou (3) indique un résultat contraire à la prévision initiale du locuteur évoquée préalablement dans l'énoncé. Par exemple, dans (2), on imagine que le locuteur s'attendait, au moins au départ, à ce que Jean n'échouerait pas à son baccalauréat compte tenu des « circonstances d'alors » $(\mathrm{A} 1)^{3}$ : par exemple, son application à préparer l'examen, les cours particuliers de perfectionnement offerts par ses parents. Il y a eu un retournement de circonstance. Si «le fait exprimé dans l'énoncé » $(\mathrm{X})$ représente le résultat contraire à ce qu'attendait le locuteur au départ, c'est vraisemblablement parce que X est qualifié par Y (A2 : circonstance malheureuse / circonstance miraculeuse), représentant une circonstance qui se co-détermine tensivement avec A1 (circonstance « d'alors » c'est-à-dire initiale).

Il est significatif que, dans ce premier cas de figure, A2 représente un état de fait qualitativement très décalé par rapprot à $\mathrm{A} 1$. Nous pouvons penser que cette caractéristique est due à leur co-détermination tensive.

\subsubsection{Deuxième cas de figure : A1 et $A 2$ sont des occurrences en succession relativement à la visée}

\subsubsection{Les emplois dits « distributifs »}

(4) Eric voit 2 clients par jour.

$\mathrm{X}$ est le procès « voir 2 clients ». $\mathrm{Y}$ « chaque jour » est ici compris comme unité temporelle pour réaliser le procès « voir 2 clients », et envisagé du point de vue d'une entité plus grande qui comporte plusieurs occurrences successives de jour ${ }^{4}$. Ce qui est illustré par l'emploi de par et de de dans la publicité d'un cybercafé : Connexion : 0.15 euros par minute ou 7.35 euros de l'heure. Relativement au « coût de l'utilisation d'internet »

\footnotetext{
${ }^{3}$ Ces caractéristiques n'apparaissent pas forcément si l'on remplace les expressions en question ici par les expressions pour son malheur; pour son bonbeur.

${ }^{4}$ Ce qui diffère d'une expression telle que 3 km à l'heure.
} 
$(\mathrm{P})$, on envisage facilement de se connecter pendant plusieurs minutes mais pas pendant plusieurs heures. D'où l'emploi de par minute mais pas de par heure quand il s'agit de la «connexion à l'internet» (X). Une autre caractéristique de l'emploi de par en question est que la 'visée' (P) est fondée / se réalise par l'interaction entre des occurrences en succession mais non pas par la simple itération d'occurrences. Par exemple, chaque occurrence de 2 clients doit être qualitativement renouvelable : on ne peut pas dire *Eric voit deux clients par jour, Luc et Guy, alors que l'on peut dire Eric voit deux clients chaque jour, Luc et Guy. Nous pouvons donc penser que, dans (4), le fait de "voir 2 clients » $(\mathrm{X})$ «chaque jour » $(\mathrm{Y})$ représente par exemple la tâche de « l'exécution du métier (quotidien) » (P), l'exécution du métier étant fondée sur la succession constante entre «le jour où la tâche est effectuée » (A1) et «le jour suivant où la tâche (d'une même extension) est à effecuer comme A1 » (A2) ${ }^{5}$. C'est-à-dire que, dans cette succession, relativement à $\mathrm{P}, \mathrm{A} 1$ et $\mathrm{A} 2$ s'interactivent par leur relation co-déterminatoire tensive. Dans (4), $\mathrm{X}$ représente l'unité de tâche attribuée à $\mathrm{Y}$ « chaque jour». L'interaction tensive entre $\mathrm{A} 1$ et $\mathrm{A} 2$ relativement à $\mathrm{P}$ expliquerait vraisemblablement pourquoi l'emploi de par en question ici peut exprimer aussi la réalisation d'une visée par une accumulation: Si tu veux finir ta thèse en 3 ans, il faut que tu écrives 100 pages par an, bein.

\subsubsection{L'expression du type courir par la ville, marcher par la chambre}

L'emploi suivant, considéré comme démodé, manifeste une forte ressemblance avec l'emploi que nous venons d'analyser :

(5) Pierre a couru par la ville.

(6) La marquise marchait par la chambre.

La première caractéristique de ce type d'exemple est que plusieurs séquences de procès s'effectuent partout sur la surface de l'espace exprimé par le régime de par (la ville / la chambre). Par exemple, dans (5), Pierre "effectue plusieurs occurrences du procès courir» $(\mathrm{X})$ sur la globalité de l'espace ville. Ici, la ville est conçu comme «l'ensemble des trajets découpés comme constituants » $(\mathrm{Y})$. La deuxième caractéristique de cet emploi est qu'il évoque souvent une situation spéciale. Par exemple, dans (5) on imagine que Pierre cherchait quelqu'un, et dans (6), la marquise avait un souci et voulait trouver une solution. Bref, avec cet emploi de par, le prédicat exprime une visée (B. Hamma 2003). Définissons respectivement la visée (P) implicite comme «trouver la personne concernée » et «trouver une solution pour enlever l'inquiétude ». Dans (5), Pierre effectue d'abord un trajet en espérant trouver la personne concernée. Puisque cette visée n'a pas été réalisée au cours de ce trajet, il passe à un autre trajet en souhaitant cette fois-ci encore réaliser la visée. De même, dans (6), la marquise continue à faire un nouveau trajet puisqu'elle n'a pas pu trouver une solution adéquate pendant qu'elle marchait, effectuant le trajet précédent. Autrement dit, deux occurrences en succession sont représentables comme suit: «trajet effectué mais au cours duquel la visée n'est pas réalisée (donc il faut passer au trajet suivant) » (A1) et « trajet, compte tenu de A1, à effectuer pour réaliser la visée » (A2). Ainsi, relativement à la visée (P), A1 et A2 sont co-déterminatoires tensivement. Notons que X (effectuer plusieurs occurrences du

\footnotetext{
${ }^{5}$ Alors qu'avec Eric voit 2 clients chaque jour, il n'y a pas d'aspect constructif entre des occurrences.
} 
Yukiyo Homma

procès courir ou marcher) est représenté par le verbe courir ou marcher. Quant à Y (l'ensemble des trajets, découpés comme constituants), représenté par le régime de par, exprime implicitement à la fois A1 et A2.

2.2.2.3. N1 par $\mathrm{N} 2$ : Vendre sa bibliothèque livre par livre; un par un; deux par deux

Il a vendu son écurie cheval par cheval. (A.-M. Berthonneau 1989)

Dans (7), contrairement au cas précédent, l'entité comportant A1 et A2 est exprimée dans la proposition principale par le syntagme son écurie, et A1 et A2 sont représentés respectivement par N1 et par N2 dans la forme N1 par N2. Dans (7), on a l'impression que la vente s'effectue petit à petit, étape par étape, en pensant, à chaque vente, au nombre de chevaux vendus ou/et au nombre de ceux qui restent jusqu'à « la vente totale de son écurie» $(\mathrm{P}, \text { la visée })^{6}$. Donc, relativement à $\mathrm{P}$, la succession d'occurrences cheval est conçue comme le contraste constant entre « (encore un) cheval vendu, qui représente une des étapes dans la gradation de la vente totale de l'écurie » (A1) et « cheval suivant à vendre, cheval qui représente l'étape suivante » (A2). Comme nous l'avons expliqué à propos du cas de Sa journée commence par la lecture, A1 et A2, étant deux étapes successives, s’impliquent sémantiquement tout en occupant un statut iremplaçable par d'autres étapes. Donc A1 et A2 sont en relation co-déterminatoire tensive relativement à $\mathrm{P}$. Notons que dans (7), X correspond à « chaque occurrence du procès vendre» et $\mathrm{Y}$, à «chaque cheval». La même explication est valable pour l'expression un(e) par un(e):

(8) Jean a analysé les exemples / Anne a examiné les produits) un par un.

Dans (8), Y est «chaque occurrence de l'entité », exemple ou produit. Dans cet exemple, la visée ne consiste pas simplement à «analyser» $(\mathrm{X})$ chaque exemple ou à « examiner» (X) chaque produit. Avec Jean a analysé les exemple un par un, on a l'impression que Jean a essayé d' "extraire un principe » $(\mathrm{P})$ en portant attention à la corrélation entre le résultat de chaque examen d'exemples. Ensuite, avec Anne a examiné les produits un par un, on conçoit qu'Anne « veille, par exemple, à ce que tous les produits n'aient pas de défaut » $(\mathrm{P})$. Donc, les deux produits en succession sont représentables comme "produit examiné et qui n'a pas de problème (ce qui permet de passer à l'examen suivant) » (A1) et « produit à examiner pour savoir s'il n'a pas de problème comme ce fut le cas pour le précédent» (A2). Bref, ici encore, nous constatons une relation codéterminatoire tensive entre deux occurrences successives. Finissons cette partie avec l'analyse de l'expression type deux par deux :

Tout le monde, en rang, deux par deux!

La visée consiste à ce que «l'ensemble des membres du groupe se mette en rang » $(\mathrm{P})$. X est « produire chaque rang ». Pour produire « un nouveau rang » (A2), on se réfère au « rang qui le précède » (A1), rang qui est déjà constitué et qui sert de référence à A2. Autrement dit, A2 ne peut exister que compte tenu de A1. Egalement du point de vue de A1, il doit tenir compte de A2 car, compte tenu de $\mathrm{P}$, on vise la suite

${ }^{6}$ Ce n'est pas le cas avec Il a vendu son écurie cheval après cheval. 
de la production des rangs. Ainsi, A1 et A2 sont réciproquement indispensables pour réaliser la visée. Les exemples suivants confirment cette explication :

(10) Plantez ces fleurs deux par deux.

(11) ??Répartissez ces fleurs dans ces vases cinq par cinq.

(12) ??Tu peux mettre ces bonbons dans les sachets trois par trois?

Dans (10), la plantation de ces fleurs par couple ne s'effectue qu'en se référant aux couples de fleurs qui les précèdent et qui sont déjà plantées. En revanche, dans (11) et (12), il s'agit de la répartition d'objets du procès dans des récipients (plateau; vases; sachets) déjà individuellement présents : impossible de concevoir la relation entre une occurrence en tant que référence (A1) et l'occurrence qui apparait en se référant à l'occurrence précédente. D’où l'échec de la relation co-déterminatoire. Passons à un autre cas de figure.

\subsubsection{Troisième cas de figure: $Y$ représente $A 1$ ou A2, un des éléments d'une même série}

\subsubsection{Lieu de passage, Instrument, Intermédiaire}

Plus haut, à propos de l'exemple passer par Lausanne, nous avons dit que relativement à $\mathrm{P}$ 《 atteindre le lieu visé », s'établit la relation co-déterminatoire tensive entre «lieu de passage à non valider» (A1) et «lieu de passage à valider» (A2, qui est ici Y, Lausanne). Dans cet exemple, A1 est implicite. Or ce n'est pas le cas dans l'exemple suivant :

\section{(13) Il ne faut pas juger d'un homme par ce qu'il ignore, mais par ce qu'il sait.}

On vise à « juger correctement d'un homme» $(\mathrm{P})$. $\mathrm{X}$ est le procès « juger d'un homme ». «Ce qu'il ignore » représente Y1, et «ce qu'il sait» Y2. En (13), l'énoncé exprime que, relativement à la visée, une relation de co-déterminatoire tensive est établie entre $\mathrm{Y} 1$ et $\mathrm{Y} 2$, Y1 représentant « l'élément à ne pas valider pour X » (A1) et Y2, «l'élément à valider pour X » (A2). Cette tension est manifestée dans l'exemple Jean a été renversé par un camion et l'exemple (13) par le contraste entre 'choix qui valide $\mathrm{X}$ ' et 'choix qui ne valide pas X'. Mais dans l'exemple suivant, la tension entre A1 et A2 est exprimée d'une autre manière, à savoir par des adjectifs oppositifs :

\section{(14) Il est petit par la taille mais il est grand par l'esprit.}

Ici, la visée $(\mathrm{P})$ est « la qualification d'une personne». Définissons comme Y1 « la taille » et comme Y2 «l'esprit». X est la personne en question dans l'énoncé. La personne $(\mathrm{X})$ est qualifiée par la tension entre $\mathrm{Y} 1$ et $\mathrm{Y} 2$, domaines co-définitoires concernant une personne. Y1 et Y2 représentent respectivement A1 et A2. L'apparition des deux qualificatifs petit et grand, qui s'interdéfinissent, est un indice de la relation co-déterminaoire entre A1 et A2. Etayons notre hypothèse par les exemples suivants :

$$
\text { ?Il est petit par la taille. }
$$

(16) Il est grand par l'esprit. 
Yukiyo Homma

L'acceptabilité différente entre (15) et (16) est explicable comme suit: quand il s'agit de «la qualification d'une personne » $(\mathrm{P})$, les adjectifs petit ou grand ont a priori une grande affinité avec la taille. L'exemple (16) ne pose pas de problème car étant donné l'affinité implicite entre l'adjectif grand et la taille, quand cet adjectif grand est porté sur un domaine, l'esprit, qui se co-détermine avec la taille par des propriétés discriminatoires, l'énoncé peut exprimer implicitement «Il est petit par la taille». Donc l'énoncé permet de rappeler la relation co-déterminatoire entre A1 et A2 pour qualifier $\mathrm{X}$ (la personne). En revanche, (15) n'est pas très acceptable car, justement, à cause de l'affinité entre l'adjectif petit et la taille, l'énoncé ne peut pas exprimer clairement le domaine dans lequel il est grand, d'où l'échec de l'établissement de la tension entre A1 et A2 pour définir X. Achevons cette partie par l'exemple suivant :

\section{Cette ville se situe par $41,5^{\circ} \mathrm{C}$ de latitude Nord et par $30^{\circ} \mathrm{C}$ de longitude Est.}

Il s'agit de « définir la localisation de cette ville sur la Terre » $(\mathrm{P})$. X est « la position de cette ville ». A1 (Y1) et A2 (Y2) sont respectivement $« 41,5^{\circ} \mathrm{C}$ de latitude Nord et $30^{\circ} \mathrm{C}$ de longitude Est $\%$. A1 et A2 sont deux éléments indispensables pour définir la position de cette ville sur la Terre, donc X. D'où la vision globale de la Terre, ce qui n'est pas forcément le cas quand on remplace par par à.

\subsubsection{Quatième cas de figure: Y représente une estimation subjective, $A 2$, qui se réfere à $A 1$}

\subsubsection{L'expression par trop}

L'adverbe trop indique un haut degré (Il est trop mignon cet enfant.) présenté comme le dépassement d'une norme ou de ce qui s'observe habituellement; si on le modifie avec par, par trop implique un jugement négatif tel que " exagérément », «vraiment trop » :

(18) Patrick est trop (beau / intelligent).

(19) Patrick est par trop (beau / intelligent).

Dans (18), il s'agit d'un dépassement du degré 'normal', ordinairement observé, mais qui ne donne pas lieu nécessairement à une désapprobation ou à la crainte de conséquences préjudiciables; on pourrait enchaîner : Patrick est trop (beau / intelligent) : je veux l'épouser! En revanche, dans (19), de la négativité est portée sur 'Patricke est (beau / intelligent) $(\mathrm{X})$ alors que l'adjectif ne dégage pas en soi de négativité. Il ne s'agit donc plus de la simple affirmation d'un excès du degré 'normal'. Dans (19), Y (trop) est considéré comme un degré non convenable (A2) parce que le locuteur calcule ce degré en se basant sur le degré de référence (A1), établi au préalable, considéré subjectivement comme 'acceptable' ou 'appropriée' opposition à laquelle A2 se définit. X est « l'être Patrick». Vu la négativité portée sur $\mathrm{A} 2$, nous pouvons penser que, relativement à la visée «jugement d'acceptabilité de degré » (P), Y (A2) détermine $\mathrm{X}$ en s'opposant à A1.

${ }^{7}$ Par n'est pas acceptable dans les cas suivants car l'état de fait qui y est exprimé ne permet pas d'indiquer le degré de référence qui servirait à porter une éventuelle estimation sur Y : *Il fait par trop beau. $/{ }^{*}$ Il est par trop bon, ce gâteau. $/{ }^{*}$ Le poulet ne cuit pas bien par $40{ }^{\circ} \mathrm{C}$. 


\subsubsection{L'expression du type par trois fois}

Une analyse semblable peut être faite de la comparaison entre trois fois et par trois fois. Un énoncé tel que Vincent est intervenu trois fois est purement descriptif, et il est possible de lui enchaîner un cotexte positif : C'est bien, il prend de l'assurance ! Mais dans (20), par introduit un point de vue, marque une rupture dans le constat:

\section{(20) Vincent est intervenu par trois fois.}

La situation ici évoquée suppose en effet soit que Vincent lui-même a été obligé d'insister (parce qu'on ne l'écoutait pas, ou que son intervention ne rencontrait pas l'écho attendu, etc.), soit que celui qui rapporte (20) manifeste par là l'impuissance de Vincent à se faire entendre, ou son exaspération, etc. Implicitement, le présupposé est qu'il aurait dû suffire d'une fois pour l'intervention de Vincent : une norme par rapport à «l'intervention de Vincent » $(\mathrm{X})$ est jugée subjectivement acceptable ou normale (A1) ; Y (A2 : trois fois) simultanément se réfère à cette norme et, par le biais de par, la montre transgressée, d'où la négativité de A2. L'interprétation «Vincent est intervenu autant de fois » est due vraisemblablement à la relation de co-détermination entre A1 et A2.

\subsubsection{L'expression par terre}

L'expression par terre est figée : aucun autre nom ne peut y apparaitre pour indiquer la 'localisation spatiale', y compris lorsque le contexte référentiel s'y prêterait $a$ priori (*Il est assis par tapis / par chaise) - on peut donc penser que terre se combine avec par en raison d'une particularité qui n'est pas simplement une indication de lieu. Nous retiendrons comme indice le fait que l'expression par terre évoque souvent un dégât ou un état détrimental : (tomber / renverser qch. / traîner) par terre, etc. Dans des expressions telles que (sauter / tomber / renverser qch.) par terre, qui expriment un passage d'état, le passage de la position initiale vers le lieu représenté par terre est considéré comme un passage entre un lieu de référence (A1), jugé comme non particulier, et un lieu possédant une certaine particularité (A2) par rapport au premier. Dans des expressions qui n'expriment pas un passage d'état, telles que être assis (dormir) par terre, un état détrimental ou un dégât ne sont presque pas évoqués, mais il n'empêche que terre est conçu comme ayant une particularité (A2) par rapport à des lieux tels que chaise, banc (pour être assis), etc. ou lit (pour dormir), bref, des lieux 'normaux', pour ainsi dire définitoires, de référence (A1), pour être assis ou dormir (X). Avec l'expression par terre, la visée P est définissable comme évaluation qualitative de la localisation d'une entité en question dans l'énoncé. Nous pouvons comprendre de la même façon l'emploi suivant (exemple 21).

\subsubsection{Les expressions du type par moments, par endroits}

Examinons l'exemple suivant:

(21) Quoi? Tu n'a pas acheté un sandwich pour moi? Qu'est-ce que t'es égoïste (par moments / ?de temps en temps), hein!

Il est significatif que le fait que «tu es égoïste » $(\mathrm{X})$ est conçu comme un fait inhabituel, et saillant a priori par rapport à ce qui se passe 'd'habitude' : le locuteur considère a priori que son interlocuteur «est égoïste» $(\mathrm{X})$ momentanément alors que ce 
dernier 'n'est pas égoïste' d'habitude. Ansi, nous pouvons supposer que Y (moments) représente 'moments inhabituels' (A2). Si Y (ici, A2) est jugé 'inhabituel', c'est parce que le locuteur le réfère à l'état habituel de la personne en question, c'est-à-dire, dans 'l'habitude' (A1). Comme le cas précédent, X est qualifié par la relation codéterminatoire entre A1 et A2 établie par Y. L'exemple suivant confirme cette explication :

(22) Oui, notre relation 'mère-fille' va très bien. Ma fille m'appelle (?par moments / de temps en temps), vient me voir tous les mois.

Ici, vu le contexte, il est étrange que X (ma fille + m'appeler) soit interprétable comme un fait inhabituel. D'où la bizarrerie de l'emploi de par moments. Quant à l'expression de temps en temps, le locuteur ne conçoit pas a priori une distinction qualitative entre temps représenté dans de temps en temps et les autres temps. Avec cet emploi de par, la visée P serait définissable comme « apparition d'occurrence(s) inhabituelle(s)».

\subsection{Bilan de l'investigation}

Nous avons observé à travers des exemples divers (2.2) comment la FS de par se configure selon les contextes. Récapitulons les cas de figure de la FS attestés au moins dans ce présent article :

1) le passage entre deux états (de fait) oppositoires (A1 et A2)

- Y permet le passage entre A1 et A2 : Il a été renversépar un camion

- Y représente A2, circonstance s'opposant à la circonstance initiale : Par miracle, il a été sauvé

- Y représente $\mathrm{A} 2$, résultat contraire à la prévision initiale du locuteur : finir partinfinitif

2) A1 et A2 sont des occurrences en succession relativement à la visée

- Y représente A1 ou A2 qui est une des étapes : GN+commencer par GN / $\mathrm{GN}+$ terminer par GN

- N1 par $\mathrm{N} 2$ où $\mathrm{N} 1$ représente $\mathrm{A} 1$ et $\mathrm{N} 2$ représente $\mathrm{A} 2$ : vendre son écurie cheval par cheval / un par un / deux par deux

- Y représente l'unité d'occurrences A1 et A2 : courir 3 km par jour

- Y représente une entité constituée par la succession immédiate entre A1 et A2 : courir par la ville

3) Y représente A1 ou A2, un des éléments d'une même série

- Y est un 'choix à valider' (A2) qui s'oppose à un (des) 'choix à non valider' (A1) : passer par la Suisse

- Y représente A1 ou A2. Leur relation est manifestée à travers des qualificatifs : Il est petit par la taille mais il est grand par l'esprit 
4) Estimation subjective : $Y$ représente $A 2$, qui se réfère à $A 1$, sa référence

- par trop beau / par trois fois / par un beau matin de printemps / par moments / par terre

Il faut maintenant vérifier si et comment la FS de par se retrouve dans les expressions par ici, par là ou par là-bas.

\section{Réflexion sur l'emploi de par se combinant avec les termes ici, là, là-bas}

D’abord, il est significatif que cet emploi de par dégage l'impression que la frontière du référent de Y est floue. Nous allons prouver que, même pour cette particularité très locale, la FS de par est présente. Ensuite, la deuxième caractéristique de cet emploi est que par n'accepte ici que des déictiques tels que ici, là, là-bas comme régime. Il importe alors d'éclaircir les caractéristiques de ces termes, seulement en ce qu'elles concernent cet article. Contrairement à un terme non déictique qui permet d'identifier son référent par une notion préconstruite indépendamment du moment de l'énonciation, un terme déictique, faute de notion, désigne son référent par un autre moyen : le repérage du référent à travers le point de vue du locuteur. Dans la plupart des cas, l'identification du référent d'un déictique est possible grâce à l'implicite situationnel, l'indication d'un geste ou une précision verbale (ici, en France). Sinon, un déictique en soi ne peut pas préciser son référent. Effectivement, le déictique ici, par exemple, ne permet pas de décoder exactement de quelle entité il s'agit: son référent peut être interprété comme France, Paris, ou encore l'espace (relativement) limité où se trouve le locuteur (ou les interlocuteurs), etc. Ainsi, tant qu'il n'y a pas de précision quelconque sur son référent, un déictique oblige l'interlocuteur à chercher le référent dans l'entité spatio-temporelle censée le contenir. Ainsi, le référent d'un déictique est repéré par rapport à d'autres entités (qui l'entourent). Mais comme nous allons le voir, la version avec par diffère de la version sans par grâce à la potentialité d'un déictique de mettre en relation son référent et d'autres entités non seulement sur le plan repérationnel mais aussi sur le plan représentationnel. C'est un facteur crucial pour les combinaisons semi-figées par ici, par là et par là-bas. Commençons par les exemples suivants :

(23) (Ici /*Par ici), à Besançon, il y a beaucoup de nature.

(24) On va (là-bas /*par là-bas), entre le poteau et l'arbre?

(25) Il s'amusait (ici / par ici), près de la porte.

Ces exemples attestent que l'emploi de par a tendance à ne pas accepter un référent possédant une frontière nette. Si, en (25), les deux versions sont acceptables, le référent de $\mathrm{Y}$ (près de la porte) est conçu de façons différentes : sans par, on conçoit un endroit comme un point mais avec par, un lieu dont le contour est flou. Cependant, le fait que le référent de ici est conçu d'une façon floue dans la version avec par n'est qu'un indice, qui est dû à un facteur fondamental. Expliquons avec un autre exemple : dans un restaurant, un serveur qui indique une place à côté de lui à un client qui est encore à l'entrée lui dira: Par ici, s'il vous plaît. Dans ce contexte, cette expression est 
proche de Passez de l'espace où vous êtes à l'espace où je suis ${ }^{8}$. A priori, le serveur distingue et met en relation les deux espaces : «l'espace où se trouve le locuteur » (A2), à savoir l'espace représenté par le terme ici (avec par) et «l'espace où se trouve le client» (A1). Donc, la représentaiton du référent de ici (avec par) est définie compte tenu d'une autre entité, ce qui n'est pas le cas avec Ici, s'il vous plâ̂t, qui précise simplement une place. Ainsi, avec par, nous retrouvons la relation co-déterminatoire tensive entre ici (A2, qui est ici Y) et «une autre entité (spatiale)»(A1). Mais la frontière représentationnelle entre A1 et A2 n'est établie que subjectivement, ce qui oblige l'interlocuteur à un décodage tacite du référent de ici. Voilà pourquoi on déduit que son contour est flou. En revanche, en (23), Besançon est référentiellement autonome, présenté comme un point. On peut associer ici à à Besançon mais non par ici, qui contredit l'indépendance référentielle du lieu. L'analyse est semblable pour (24) : là-bas localise un lieu référentiellement autonome, défini par entre le poteau et l'arbre, tandis que par là-bas définit un lieu dont la référence dépend, sur le plan représentationnel, des lieux qui l'entourent (donc le référent de là-bas n'est pas autonome). Il est vrai que entre le poteau et l'arbre, contrairement à à Besançon, ne circonscrit pas un point mais une surface. Mais ce qui est constant est que son référent n'a pas la potentialité d'établir sa dépendance (continuité) représentationnelle par rapport à son entourage : c'est donc la potentialité du référent d'être autonome ou dépendant sur le plan représentationnel qui constitue le facteur déterminant des possibilités d'emploi de par dans par ici ou par là-bas.

Les exemples (26) et (27) permettent de confirmer cette hypothèse : les termes repris pour désigner le référent d'un déictique représentent une entité munie d'une frontière nette. Donc on s'attend à ce que, comme en (23) et en (24), ils ne puissent pas se voir apposer par ici ou par là-bas, mais, en réalité, cela n'empêche pas l'anaphore ou la cataphore en par. La distinction 'frontière nette / frontière floue' se trouve donc encore ici non pertinente :

$$
\text { (Ici / Par ici), en Franche-Comté, ily a beaucoup de vaches. }
$$

On va (là-bas / Par là-bas), à l'ombre?

Ainsi, les termes repris pour désigner le référent d'un déictique tolèrent aussi bien une apposition en ici / là-bas qu'en par ici / par là-bas, contrairement à Besançonc'est donc qu'ils sont susceptibles de deux interprétations. On peut par exemple considérer l'ombre en soi, comme on dit la lumière sans que ce soit forcément en opposition avec l'obscurité, ou le soleil sans le concevoir en contraste avec la lune, etc. : la mise en relation avec ici ou là-bas correspondrait à ce premier cas de figure. Ou bien, au contraire, on peut parler de lieu ombragé par co-détermination avec lieu ensoleillé : la mise en relation avec par ici ou par là-bas correspondrait à ce second cas de figure. Dans On va là-bas, à l'ombre, l'ombre est indépendant sur le plan représentationnel ; en revanche, avec On va par là-bas, à l'ombre, «lieu ombragé » (A2), et le «lieu ensoleillé » (A1) où se trouvent a priori les interlocuteurs, sont en relation de co-détermination. Voilà

\footnotetext{
${ }^{8}$ Dans un autre contexte, l'interprétation de Par ici, s'il vous plaît est proche de Suivez-moi en passant par ce lieu (pour arriver à un autre lieu). Ici, nous pouvons comprendre ce cas de la même façon que les exemples tels que passerpar la Suisse, voyager par mer.
} 
pourquoi le choix du lieu est éventuellement motivé par quelque(s) propriété(s) : Allons par là-bas, il fait plus frais / je ne supporte pas le soleil, etc.

Nous pouvons appliquer notre analyse à des expressions telles que par chez vous. La différence d'interprétation est sensible si l'on compare les exemples suivants ; la version sans par interpelle l'interlocuteur sur ce qui se passe chez lui, indépendamment de ce qui peut se passer ailleurs :

\section{(28) Ilpleut, chez vous? / Ilpleut, par chezvous?}

Mais dans la version avec par, le référent de chez vous désigne par exemple une 'région', une 'campagne', une entité plus large, dont les propriétés peuvent évoquer leur relation avec celles d'un autre lieu, en l'occurrence l'espace où se trouve le locuteur: par exemple, l'énoncé Fait-il beau, par chez vous, à la campagne? fait penser que le locuteur se trouve dans un espace urbain, c'est-à-dire un lieu dont les propriétés se codéterminent avec celles de la campagne. En réalité, l'expression ?Fait-il beau par chez vous, à Dijon ? parait étrange car à Dijon ne rappelle pas une entité spatiale avec laquelle il se co-détermine.

Ainsi, avec par, le référent de chez vous (A2) se définit par opposition à une autre entité (A1). On peut faire la même analyse d'énoncés dans lesquels Y représente une entité temporelle :

\section{Il est né en 1953 ou 1954..., par là.}

Cet énoncé est paraphrasable par Il est né à peu près en 1953 ou en 1954 ; l'entité qui est prête à être désignée par Y (lä) est présentée au préalable : c'est la marge temporelle définie par 1953 ou 1954... Le locuteur calcule cette marge (A2) par rapport à d'autres temps (A1) soit qui le précèdent soit qui le suivent, bref, à d'autres temps qui l'entourent. A2, qui est une estimation subjective, se réfère à A1. Puisque A2 est une estimation subjective, elle se définit à travers l'inter-calcul avec A1, et ne peut donc établir qu'une frontière floue avec A1. Renforçons notre hypothèse avec l'expression par le passé:

\section{(30) Ils s'entendent mieux que par le passé.}

Le passé est forcément le passé d'une autre période temporelle (ici, le moment de l'énonciation : A1), comme le père est forcément le père d'un enfant, par exemple. Ainsi, les propriétés de $\mathrm{Y}$ (A2 : le passé) sont définies par opposition à A1 (le moment de l'énonciation). C'est-à-dire que deux moments différents se co-déterminent.

\section{Conclusion}

Nous avons prouvé que l'identité de par se présente même dans des expressions considérées plus ou moins comme figées et qu'elle sous-tend l'effet de sens 'approximation' dans les expressions par icí, par là et par là-bas. Nous avons démontré que, comme dans les autres emplois, cet effet de sens est le résultat de l'interaction entre les éléments du co-texte. Ces expressions paraissent comme (semi-)figées, parce que, d'abord, le contexte ne permet à la FS qu'un seul type d'interaction, de réalisation, à savoir la combinaison 'par + déictique'. Une autre raison serait qu'avec l'emploi du 
type par ici, notre cognition n'arrive pas à concevoir l'effet de sens (ici, l'approximation) en dissociant par et le déictique, d'autant plus que l'on sait que par n'exprime pas en soi une approximation.

Ce que nous avons proposé comme la FS de par n'est pas facilement identifiable à partir de l'effet de sens 'approximation'. Si, maintenant, on compare cet effet de sens et la FS de par que nous avons proposée, on se rend compte à quel point notre perception immédiate peut être trompeuse.

\section{BIBLIOGRAPHIE}

Berthonneau, A.M. (1989), Composantes linguistiques de la références temporelle, thèse d'état, Université Paris VII.

CADIOT, P. (1997), Les prépositions abstraites en français, Armand Colin.

Culioli, A. (2000), Pour une linguistique de l'énonciation, Tome 1, Paris, Ophrys.

Franckel, J.J., Paillard D., Saunier E. (1997), «Modes de régulation de la variation sémantique d'une unité lexicale. Le cas du verbe PASSER», dans La locution, collection 'Saint-Cloud', Klincksieck, Paris, pp. 49-68.

Hamma, B. (2003), «Y a-t-il une continuité entre par- préfixe et par préposition ? L'exemple de Parcourir ", Actes des 6ème RJC Langage et langues, Paris III, Presses Universitaires de la Sorbonne, pp. 65-69.

Homma Y. (2002-2003), Approche énonciative des emplois de la préposition PAR, mémoire de DEA Sciences du Langage, dir. Daniel Lebaud, Université de Franche-Comté, Besançon.

LEeman, D. (1995), "Pourquoi peut-on dire Max est en colère mais non*Max est en peur? Hypothèses sur la construction être en $N »$, Langue française, 105, Paris, Larousse, pp. 5569.

OGuma, K. (2003), « Peut-on saisir la polysémie de PAR à travers la représentation spatiale ?», Exemplier commenté de la Conférence de l'Association savante japonaise sur la linguistique française, le 18 octobre 2003, Tokyo.

PAILlard, D. (1995), "Perestroïka, à propos du préverbe pere- en russe contemporain », in Langues et langage. Problèmes et raisonnement en Linguistique, Mélanges offerts à Antoine Culioli, Presses Universitaires de France, 1995, Paris, pp. 345-357.

KWON-PAK, S.N. (1997), Les prépositions spatiales : sur quelques emplois de PAR, thèse de doctorat, Université des Sciences Humaines de Strasbourg.

STOSIC, D. (2002), PAR' et 'A TRAVERS' dans l'expression des relations spatiales : comparaison entre le français et le serbo-croate, thèse de doctorat, Université de Toulouse-Le Mirail. 